\title{
CIRCLE PACKINGS IN THE APPROXIMATION OF CONFORMAL MAPPINGS
}

\author{
KENNETH STEPHENSON
}

Connections between circle packings and analytic functions were first suggested by William Thurston [T2], who conjectured that the conformal mapping of a simply connected plane domain $\Omega$ to the unit disc $\Delta$ could be approximated by manipulating hexagonal circle configurations lying in $\Omega$. The conjecture was confirmed by Rodin and Sullivan [RS]. Their proof relies heavily on the hexagonal combinatorics of the circle configurations, a restriction not suggested by the underlying intuition.

The purpose of this note is to announce that Thurston's conjecture is true with much weaker combinatoric hypotheses and to outline the proof. The main lines of argument are those developed by Rodin and Sullivan, but the proof is independent. The deepest part of their work-a uniqueness result of Sullivan's which depends on Mostow rigidity-is replaced here by probabilistic arguments. We work in the setting of hyperbolic geometry and make use of the discrete Schwarz-Pick lemma proven in [BS] to understand the behavior of circle configurations. We analyze how curvature distributes itself around a packing as successive differential changes are made to boundary circles, ultimately modelling this process as a random walk. The proof that a certain limiting random walk is recurrent replaces the uniqueness result of Sullivan. Details will appear elsewhere.

The author gratefully acknowledges support of the National Science Foundation and the Tennessee Science Alliance.

\section{Statement of THE MAIN RESUlt}

Let $P$ denote a finite collection of circles in the plane having mutually disjoint interiors. Connect centers of tangent circles with euclidean line segments. If a triangulation of a simply connected closed region of the plane results, we say that $P$ is a circle packing.

Received by the editors November 1, 1989 and, in revised form, April 23, 1990.

1980 Mathematics Subject Classification (1985 Revision). Primary 30C35; Secondary 30G25, 51M99. 
The triangulation is termed the carrier of $P, \operatorname{carr}(P)$; the associated abstract 2-complex, denoted $K$, encodes the combinatorics. Circles on the edge of $P$ are boundary circles; the rest are interior. The flower of a circle refers to its star of faces in $\operatorname{carr}(P)$. Write $\operatorname{mesh}(P)$ for the (euclidean) radius of the largest circle in $P$.

Given a circle packing $P$, a theorem of Andreev (see [T1, Chapter 13]) implies the existence of a combinatorially equivalent circle packing $\widetilde{P}$ in the unit disk $\Delta$ which has boundary circles internally tangent to the unit circle. The combinatorial equivalence means that they have the same complex; thus, there is a correspondence $c \leftrightarrow \widetilde{c}$ between circles of $P$ and $\widetilde{P}$, so that two circles are tangent in $P$ if and only if their counterparts are tangent in $\widetilde{P}$. With normalizations to be specified shortly, $\widetilde{P}$ is unique and we call it the Andreev packing for $P$. A nonhexagonal example is illustrated in Figure 1.

In the sequel, $\Omega$ will denote a simply connected open set, with $0 \in \Omega \subseteq \Delta=\{|z|<1\}$ and $0 \neq x \in \Omega$ a distinguished point. We consider circle packings $P$ lying in $\Omega$ with $0, x \in \operatorname{carr}(P)$ and normalize the Andreev packings $\widetilde{P}$ so that the circle of $P$ whose flower contains 0 corresponds to the circle of $\widetilde{P}$ centered at 0 , while the circle of $P$ whose flower contains $x$ corresponds to a circle in $\widetilde{P}$ centered on the positive real axis. Define the simplicial homeomorphism $f_{P}: \operatorname{carr}(P) \rightarrow \operatorname{carr}(\widetilde{P})$ by mapping the center of each circle $c$ of $P$ to the center of its counterpart $\widetilde{c}$ in $\widetilde{P}$ and then extending via euclidean barycentric coordinates (i.e.,. affinely on each face of $\operatorname{carr}(P))$.
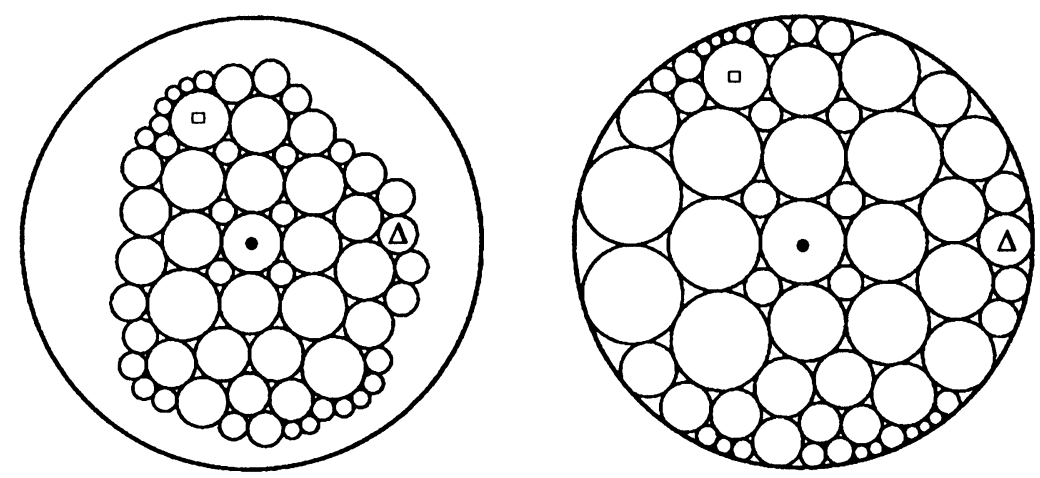

FIGURE 1 
Theorem. Let $f: \Omega \rightarrow \Delta$ be the conformal mapping with $f(0)=0$ and $f(x)>0$. Let $\left\{P_{n}\right\}$ be a sequence of circle packings in $\Omega$, with Andreev packings $\left\{\widetilde{P}_{n}\right\}$ and simplicial maps $\left\{f_{n}\right\}$ as defined above. Assume that $\operatorname{mesh}\left(P_{n}\right) \rightarrow 0$; that the sequence $\left\{\operatorname{carr}\left(P_{n}\right)\right\}$ exhausts $\Omega$; and that there exists a constant $\mathscr{C}_{\text {ratio }}<\infty$ so that the ratio of the euclidean radii of any two circles in $P_{n}$ is no greater than $\mathscr{C}_{\text {ratio }}$ for all $n$. Conclusion: $f_{n}$ converges to $f$ uniformly on compact subsets of $\Omega$.

\section{OUTLINE OF THE PROOF}

The overall strategy is that of Rodin and Sullivan: One first shows that the $f_{n}$ are uniformly $\kappa$-quasiconformal on compacta, that their domains exhaust $\Omega$, and that their ranges exhaust $\Delta$. As a consequence of quasiconformality, the $f_{n}$ are equicontinuous on compacta and form a normal family. The additional mapping conditions and normalizations ensure that the limit function $f$ of any subsequence is a $\kappa$-quasiconformal homeomorphism of $\Omega$ onto $\Delta$. This much follows, by and large, as in [RS], and we will not pursue the details here. Instead, we emphasize the

Key Fact. Given a compact set $E \subset \Omega$ and $\varepsilon>0$, there exists $N$ so that the restriction of $f_{n}$ to $E$ is $(1+\varepsilon)$-quasiconformal for $n>N$.

With this, one concludes that the quasiconformal deformation of $f_{n}$ goes to 1 on compacta as $n \rightarrow \infty$, hence that any limit function $f$ will be 1 -quasiconformal, i.e., conformal, and the proof is complete.

Essential to the proof of the key fact in [RS] is the uniqueness, due to Sullivan, of the infinite hexagonal packing in the plane: Their circle packings have complexes which are part of one infinite hexagonal complex, and faces deep inside their carriers will be approximately equilateral. In our setting, however, the combinatorics of the packings $P_{n}$ are infinitely variable-there is not a single infinite supercomplex - and we do not expect equilateral faces. We argue, instead, that for large $n$, a triangle deep in $\operatorname{carr}\left(\widetilde{P}_{n}\right)$ has approximately the same angles as the corresponding triangle in $\operatorname{carr}\left(P_{n}\right)$; approximate conformality of $f_{n}$ follows. Indeed, we work with a fixed circle packing $P$ and investigate closely what happens to an individual angle in one of the triangles of its carrier as one moves in a certain continuous fashion from $P$ to its Andreev packing $\widetilde{P}$. For triangles in a fixed compact subset of $\Omega$, 
there is a bound on the change in any such angle which depends on $\operatorname{mesh}(P)$. Moreover, this bound goes to zero as $\operatorname{mesh}(P) \rightarrow 0$, so the key fact and the theorem follow.

\section{Preliminaries}

In the sequel: $\Omega \subseteq \Delta$ is as above; $E$ is a compact subset and $\delta$ a small positive number with $E+\delta \subset \Omega$. $P$ will be a circle packing lying in $\Omega$, with $E+\delta \subseteq \operatorname{carr}(P)$. Only later do we need the additional hypotheses of the theorem.

The abstract 2-complex for $P$ (and $\widetilde{P}$ ) is denoted $K$; it has $k$ vertices, $\left\{v_{1}, v_{2}, \ldots, v_{k}\right\}$, the first $q$ being boundary vertices, the remaininig $p$ interior, $k=q+p$. We put a structure on $K$ by specifying a $k$-vector $r=\left[r_{1}, r_{2}, \ldots, r_{k}\right]$ of hyperbolic radii for the vertices, obtaining a hyperbolic complex which we denote by $K(r)$. The vector $r$ lies in the set $\mathscr{R}=(0, \infty]^{q} \times(0, \infty)^{p}$, with any infinite boundary radii corresponding to horocycles in $\Delta$.

In a hyperbolic complex $K(r)$, each face has three vertices and hence three radii from $r$. There is an essentially unique way to position three mutually externally tangent circles in $\Delta$ having these radii, and the hyperbolic triangle formed by their centers determines an angle at each vertex. In this manner the radii $r$ determine angles throughout the complex. The sum of those angles determined at a given vertex $v_{j}$ in the various faces of its star is termed the angle sum for $v_{j}$ and is denoted $\theta_{j}(r)$. We call $K(r)$ a packing if $\theta_{j}(r)=2 \pi$ for all the interior vertices $v_{j},(q+1) \leq j \leq k$.

The hyperbolic complexes $K(r)$ were studied extensively in [BS], and certain results are crucial here. For two radii vectors $r, r^{\prime} \in \mathscr{R}$, write $r \leq r^{\prime}$ if their components satisfy $r_{j} \leq r_{j}^{\prime}, 1 \leq$ $j \leq k$.

Lemma 1. Assume that the complex $K$ is simplicially equivalent to a finite triangulation of a closed disc. Then:

(a) (Andreev's Theorem) There exists a unique vector $\widetilde{R}$ of radii so that $K(\widetilde{R})$ is a packing and $\widetilde{R}_{j}=\infty, 1 \leq j \leq q$; that is, every boundary radius is infinite. (Note that $K(\widetilde{R})$ is the hyperbolic complex associated with the Andreev packing $\widetilde{P}$ for $K$.)

(b) (Dirichlet Problem) Given boundary radii $r_{j}, 0<r_{j} \leq \infty$, $1 \leq j \leq q$, there exists a unique collection of interior radii so that the aggregate $r$ makes $K(r)$ a packing. 
(c) (Monotonicity) If $K(r)$ and $K\left(r^{\prime}\right)$ are packings with $r_{j} \leq r_{j}^{\prime}$, $1 \leq j \leq q$, then $r \leq r^{\prime}$ and $\theta_{j}(r) \geq \theta_{j}\left(r^{\prime}\right), 1 \leq j \leq q$. (That is, larger boundary radii imply larger interior radii and smaller boundary angle sums.) $\widetilde{R}$.

(d) (Discrete Schwarz Lemma) If $K(r)$ is any packing, then $r \leq$

\section{THE PARAMETERIZATION}

We use the radii vectors of $\mathscr{R}$ to parameterize the structures on $K$. Write $R=\left[R_{1}, \ldots, R_{k}\right]$ (resp. $\widetilde{R}=\left[\widetilde{R}_{1}, \ldots, \widetilde{R}_{k}\right]$ ) for the vector of radii associated with $P$ (resp. $\widetilde{P}$ ). Our aim is a certain smooth path of structures, starting from $K(R)$ and ending with the Andreev packing $K(\widetilde{R})$.

Define $\Theta: \mathscr{R} \rightarrow \mathbf{R}^{k}$, mapping the $k$-vector of radii $r$ to the $k$-vector of angle sums by $\Theta(r)=\left[\theta_{1}(r), \theta_{2}(r), \ldots, \theta_{k}(r)\right]$ and write $\mathscr{A}=\Theta(\mathscr{R})$ for its range. We make several observations about $\Theta:$ (1). $\Theta$ is smooth. (2). $\Theta: \mathscr{R} \rightarrow \mathscr{A}$ is one-to-one (by the Perron argument in [BS]) and invertible. (3). $K(r)$ is a packing iff $\theta_{j}(r)=2 \pi,(q+1) \leq j \leq k$. (4). The vector $\widetilde{R}$ of Andreev radii is the unique vector for which $K(\widetilde{R})$ is a packing with boundary angle sums $\theta_{j}(\widetilde{R})=0,1 \leq j \leq q$.

Let $\mathscr{S} \subset \mathscr{R}$ denote the collection of vectors $r$ for which $K(r)$ is a packing and let $\mathscr{B} \subset \mathscr{A}$ be the associated vectors of angle sums, $\mathscr{B}=\Theta(\mathscr{S})$. Note that $\mathscr{B}$ is the intersection with $\mathscr{A}$ of the $q$-dimensional affine space of vectors in $\mathbf{R}^{k}$ whose last $p$ coordinates are $2 \pi . \mathscr{S}$ is a $q$-dim smooth submanifold of $\mathscr{R}$, with $R, \widetilde{R} \in \mathscr{S}$. Let $\lambda:[0,1] \rightarrow \mathscr{B}$ be the line segment defined by $\lambda(t)=t \Theta(\widetilde{R})+(1-t) \Theta(R)$. If we define $r:[0,1] \rightarrow \mathscr{S} \subset \mathscr{R}$ as the composition $\Theta^{-1} \circ \lambda$, then $r(t)$ is a smooth simple path in $\mathscr{S}$ starting at $R$ and ending at $\widetilde{R}$; the map $t \mapsto K(r(t))$ is the desired continuous evolution of packings from $K(R)$ to $K(\widetilde{R})$.

We use differentials to estimate the quantities of interest as we move along this path. $d \lambda$ is the constant vector $d \lambda=\left[-\varnothing_{1} d t, \ldots\right.$, $\left.-\varnothing_{q} d t, 0, \ldots, 0\right]$, where $\varnothing_{j}=\theta_{j}(R), 1 \leq j \leq q$. If $\partial \Theta / \partial r=$ $\left[\left(\partial \theta_{i} / \partial r_{j}\right)\right]$ denotes the Jacobian matrix of $\Theta$, then the differential $d r$ of our path is the vector satisfying

$$
\left(\frac{\partial \Theta}{\partial r}\right) \cdot d r^{\mathrm{tr}}=d \lambda^{\mathrm{tr}}
$$

The computations can be done using a Markov chain model. How- 
ever, let us set up the precise objective: Denote by $K_{E}$ the subcomplex of $K$ associated with faces in $\operatorname{carr}(P) \cap E$. Fix a face $F$ of $K_{E}$ and assume that $v_{1}, v_{2}$, and $v_{3}$ are its vertices. Each vector $r(t)$ of radii for $K$ determines a hyperbolic triangle corresponding to $F$ and thereby an angle $\psi(t)$ at $v_{1}$. In particular, $\psi(0)$ is the angle at $v_{1}$ in $\operatorname{carr}(P)$, while $\psi(1)$ is the corresponding angle in $\operatorname{carr}(\widetilde{P})$, and $|\psi(1)-\psi(0)| \leq \int_{0}^{1}|(d \psi / d t)| d t$. Suppose we prove

Lemma 2. $\int_{0}^{1}|(d \psi / d t)| d t \leq \mathscr{C}[\operatorname{mesh}(P)]$, where $\mathscr{C}$ is a constant independent of $P$ and $v_{1} \in K_{E}$.

Then, when $\operatorname{mesh}\left(P_{n}\right)$ is very small, the angles in those faces of $P_{n}$ lying in $E$ will not change much in going from $P_{n}$ to $\widetilde{P}_{n}$. This clearly implies that the simplicial map $f_{n}$ will not distort these faces much, and the key lemma follows.

\section{Modelling with a Markov Process}

In this final section, we describe briefly the Markov chain model which leads to a proof of Lemma 2, emphasizing the underlying intuition. Our task splits naturally into two parts: In the first, we continue the study of structures on a fixed $K$, showing how to compute $d r$ and $d \psi$. In the second, we see how the hypotheses of the theorem enter as mesh size goes to zero.

To begin, then, $K$ is a fixed complex as before; we find ourselves at a point $r(t)$ of $\mathscr{S}$. The angle $\psi(t)$ we are watching depends on $r(t)$, so we want to compute the differential $d r$ for which (1) holds. By Lemma $1(b)$, differentials of boundary radii determine those of the interior. Our plan is to modify boundary radii (in a differential sense) to achieve the desired angle sum decreases $d \lambda$ at boundary vertices, and then to watch as the effects of these changes ripple through the complex in a sequential fashion. We rely on repeated use of an elementary geometric fact:

Fact. An increase in radius at a vertex will decrease the angle sum there, but will increase the angle sums at neighboring vertices.

Here is the process: To decrease the boundary angle sums, we need to increase boundary radii. Start by making the appropriate increases. Any vertex neighboring a boundary vertex will see one or more neighbors growing and hence will see its angle sum increasing. It will then feel a need to increase its radius, either to reestablish an angle sum of $2 \pi$ if it is an interior vertex or to preserve its (new) angle sum if it is a boundary vertex. Assume once more that we make appropriate adjustments in radii. Now, 
the neighbors of these vertices, seeing this growth, will feel upward pressure on their radii. Make the necessary increases again. Then the neighbors of these feel upward pressure, and so on and so forth ad infinitum. Fortunately, this process finally stops, for in addition to putting upward pressure on its neighbors, each radius change also contributes to the hyperbolic area of its faces-thus as the effects of our original changes at the boundary ripple from neighbor to neighbor, they eventually dissipate entirely into area. At the end of the day, one looks at each radius to see the accumulated increases it has undergone. These changes comprise the vector $d r$ of radius differentials.

Another elementary feature of hyperbolic geometry explains why this rippling process may be modelled as a random walk: Consider what happens at an interior vertex $v_{j}$ having $m$ neighbors in the scenario above. The angle sum $\theta_{j}$ is $2 \pi$, so its star of faces is a hyperbolic polygon with $m$ sides and $m$ interior angles, say $\alpha_{n}, \quad 1 \leq n \leq m$. The hyperbolic area, $A_{j}$, of this polygon is $A_{j}=\pi(m-2)-\sum_{n} \alpha_{n}$, implying

$$
A_{j}+\sum_{n=1}^{m} \alpha_{n}=m \pi-\theta_{j}
$$

Suppose, now, that there were a small excess (above $2 \pi$ ) in the angle sum at $v_{j}$, as would result if a neighbor had recently increased in radius. We refer to this differential excess as curvature-think of it as a small quantity of angle. A small increase in $r_{j}$ will return $\theta_{j}$ to $2 \pi$. What happens to the curvature? Well, the angles $\alpha_{n}$ will each increase slightly, so part of the curvature shifts to the neighbors, resulting in small excesses in each of their angle sums. But the area $A_{j}$ will also increase, meaning that part of the curvature is absorbed as area. Curvature absorbed as area will stay put and can cause no further adjustments, whereas curvature distributed to the neighbors will necessitate increases in their radii, continuing the ripple effect of the process. Why is this a random walk? By the identity (2), $100 \%$ of the differential changes in $\theta_{j}$ are accounted for in differential changes to the angles $\alpha_{n}$ of neighbors and in the differential change to area $A_{j}$. This, in fact, suggests the transition probabilities: The transition probability from $v_{j}$ to a neighbor $v_{i}$ is the proportion of curvature at $v_{j}$ which gets shuttled to $v_{i}$ when we adjust the radius at $v_{j}$. The remainder of the curvature, called leakage, contributes to an increase in the area $A_{j}$. 
In summary, then, the complex $K$ is treated as a network, very much like an electrical circuit. Curvature is pumped in via the initial (differential) changes in boundary radii and distributes itself about the network as a random walk, propelled by changes in radii. The curvature ultimately leaks to ground (is absorbed as area) at the various vertices, and the process stops. In the language of Markov chains, the vector $-d \lambda$ serves as an initial distribution of curvature; from the Markov transition matrix one can compute precisely where curvature is absorbed; and it is then a simple matter to compute the associated vector $d r$. It should be emphasized that this model is not an approximation of how angle differentials propagate about the complex, but is absolutely precise, reflecting the geometric rigidity of circle packings.

And how does this all affect the angle $\psi$ of central interest? Recall from above that $\psi$ depends only on the radii of $v_{1}, v_{2}$, and $v_{3}$, that is, only on components $d r_{1}, d r_{2}$, and $d r_{3}$ of $d r$. These increments are all positive; however, $\psi$ is monotone decreasing in $r_{1}$ and monotone increasing in $r_{2}$ and $r_{3}$. Even if all the radii were small and comparable, a disproportionate change in one could have a large effect on $\psi$. This brings us to the second part of our description-namely, what happens as $\operatorname{mesh}(P)$ goes to zero. Here the hypothesized properties on our packings enter, yielding important additional details about the associated Markov processes.

Specifically, we need to understand the distribution, call it $U$, of all the curvature after the random walk. The study of $U$ develops roughly as follows: Transition probabilities for our Markov process can be computed using conductances, which not only proves that the process is reversible, but also gives explicitly an associated equilibrium distribution $\pi$. The function $V=U / \pi$ is found to be a nonnegative discrete harmonic function $V$ on the network $K$. The crucial estimates follow from a discrete analogue of the classical Harnack inequality for such functions; essentially, one shows that $\left|1-V\left(v_{j}\right) / V\left(v_{i}\right)\right|$ is small for neighboring vertices $v_{j}, v_{i} \in K_{E}$. Bounds are obtained via a succession of comparisons of our random walk to others, and in these the constant $\mathscr{C}_{\text {ratio }}$ in the hypotheses of the theorem enters. An important distortion theorem proven via the discrete Schwarz-Pick lemma of $[\mathrm{BS}]$ gives a bound on the factor by which circles of $P$ centered in $E$ can grow 
as the radii move toward their Andreev values $\widetilde{R}$ :

Lemma 3. There exists a constant $\mathscr{C}$ depending only on $\delta$ and $\mathscr{C}_{\text {ratio }}$ such that $1 \leq \widetilde{R}_{j} / R_{j} \leq \mathscr{C}$ whenever $v_{j} \in K_{E}$.

This gives some uniformity on conductances and allows use of the notions of "irrelevance of bounded twiddling" and " $k$-fuzz" [DSr, Chapter 8] to compare our random walk on $K$ to the simple random walk on an infinite square lattice. The latter is known to be recurrent by a famous result of Polya. The upshot of the comparisons is an estimate of escape probabilities from vertices of $K_{E}$ which establishes the desired Harnack inequality.

We can now suggest intuitively what happens as $\operatorname{mesh}(P)$ gets small: The leakage probabilities in the random walk are comparable to $[\operatorname{mesh}(P)]^{2}$; with $\operatorname{mesh}(P)$ small, a quantum of curvature will make many transitions from vertex to vertex in its random walk before leaking to area. This suggests, via the Harnack inequality, that the final distribution $U$ of curvature comes to approximate the equilibrium distribution $\pi$ of the process. Now, a very pleasing little identity (linking hyperbolic geometry, conductances, and transition probabilities) shows that if the curvature were distributed exactly as $\pi$, the competing effects of radius changes on $\psi$ would precisely balance, giving $d \psi=0 . U$ approximates $\pi$ sufficiently closely that an integration on $t$ and an appeal to Lemma 3 completes the proof of Lemma 2.

\section{REFERENCES}

[BS] A. F. Beardon and K. Stephenson, The Schwarz-Pick lemma for circle packings, preprint.

[DS] P. G. Doyle and J. L. Snell, Random walks and electrical networks, The Carus Math. Monographs, Math. Assoc. America, Washington, D.C., 1984.

[RS] B. Rodin and D. Sullivan, The convergence of circle packings to the Riemann mapping, J. Differential Geom. 26 (1987), 349-360.

[T1] W. Thurston, The geometry and topology of 3-manifolds, preprint, Princeton Univ. Notes.

[T2] _ The finite Riemann mapping theorem, Invited talk, An International Symposium at Purdue University on the occasion of the proof of the Bieberbach conjecture, March 1985.

University of Tennessee, Department of Mathematics, Knoxville, TENNESSEE 37996-1300 
